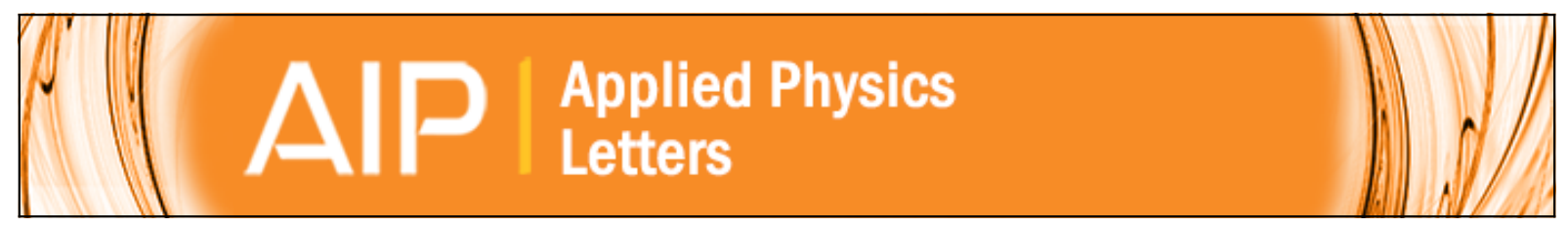

\title{
Optical coherence computed tomography
}

Li Li and Lihong V. Wang

Citation: Applied Physics Letters 91, 141107 (2007); doi: 10.1063/1.2793625

View online: http://dx.doi.org/10.1063/1.2793625

View Table of Contents: http://scitation.aip.org/content/aip/journal/apl/91/14?ver=pdfcov

Published by the AIP Publishing

\section{Articles you may be interested in}

Region-of-interest diffuse optical tomography system

Rev. Sci. Instrum. 87, 013701 (2016); 10.1063/1.4939054

A multi-view time-domain non-contact diffuse optical tomography scanner with dual wavelength detection for intrinsic and fluorescence small animal imaging

Rev. Sci. Instrum. 83, 063703 (2012); 10.1063/1.4726016

Diagnostic efficacy of computer extracted image features in optical coherence tomography of the precancerous cervix

Med. Phys. 38, 107 (2011); 10.1118/1.3523098

Solution of a Radiative Transfer Problem in a Biological Tissue. An Optical Tomography Model.

AIP Conf. Proc. 935, 237 (2007); 10.1063/1.2795420

Iterative reconstruction scheme for optical tomography based on the equation of radiative transfer

Med. Phys. 26, 1698 (1999); 10.1118/1.598661

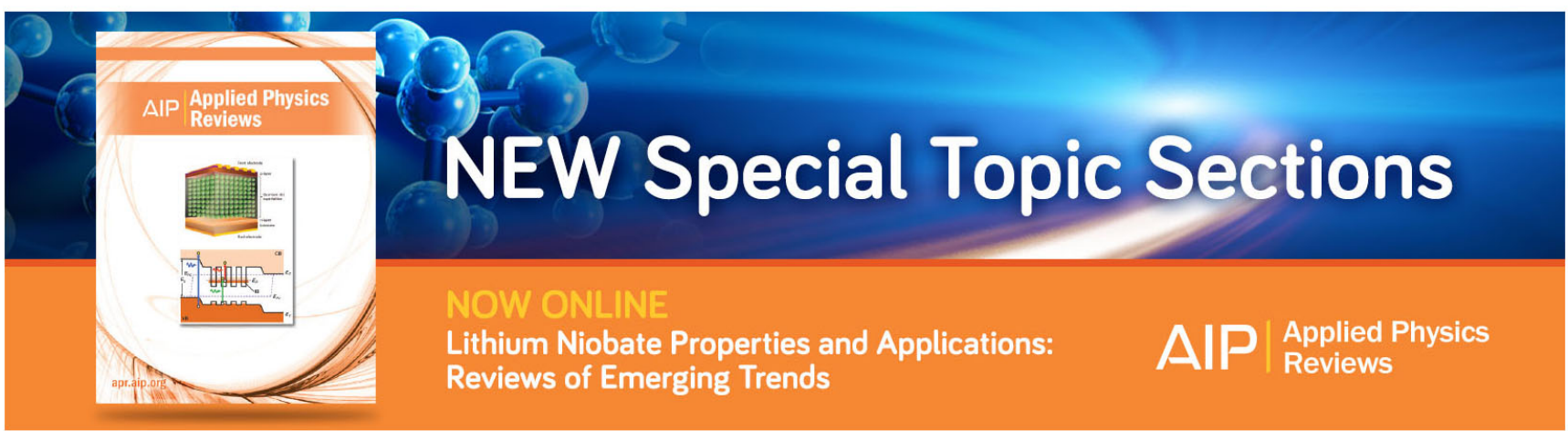




\title{
Optical coherence computed tomography
}

\author{
Li Li and Lihong V. Wang ${ }^{\text {a) }}$ \\ Optical Imaging Laboratory, Department of Biomedical Engineering, Washington University in St. Louis, \\ St. Louis, Missouri 63130, USA
}

(Received 16 July 2007; accepted 11 September 2007; published online 2 October 2007)

\begin{abstract}
A time-resolved optical tomography, optical coherence computed tomography, is proposed to bridge the gap between diffuse optical tomography and optical coherence tomography. Both ballistic and multiple-scattered photons are measured at multiple source-detection positions by low-coherence interferometry providing a temporal resolution smaller than $100 \mathrm{fs}$. A light-tissue interaction model was established using the time-resolved Monte Carlo method. The optical properties were then reconstructed by solving the inverse transient radiative transport problem under the first Born approximation. Absorbing inclusions of $100 \mu \mathrm{m}$ diameter were imaged through a 2.6-mm-thick ( 30 scattering mean-free-paths) scattering medium. (C) 2007 American Institute of Physics.
\end{abstract}

[DOI: $10.1063 / 1.2793625]$

The last decade has witnessed the revolutionary development of optical imaging techniques for biomedical applications. Among them, time-resolved techniques are known to provide the richest information on tissues' optical properties. We present a time-resolved optical imaging modality referred to as optical coherence computed tomography (OCCT or optical CCT). OCCT marries the concepts of the two most popular optical tomographical techniques-diffuse optical tomography $^{1,2}$ (DOT) and optical coherence tomography (OCT). ${ }^{3,4}$

DOT works in the diffusive regime and provides maps of tissues' optical properties through model-based reconstruction from measurements at a large number of sourcedetection pairs. It can image a few centimeters into tissue using near-infrared light. However, the spatial resolution achieved is relatively poor, typically on the order of millimeters (1/10 of the imaged depth), due to the nature of diffusion. The time-resolved DOT measures the temporal point spread function (TPSF) of the emitted light through a medium in response to an ultrashort laser pulse. It is expected to outperform the $\mathrm{cw}$ and frequency-domain versions since it carries information of the whole spectrum simultaneously. TPSFs were measured by sophisticated high-sensitivity detection systems, such as a streak camera ${ }^{5}$ and a timecorrelated single-photon counting system. ${ }^{6}$ However, the slow frame rate and the high cost limited their application. Recently, time-gated optical image intensifier ${ }^{7}$ has been utilized to develop fast parallel time-resolved DOT. However, the gate width is $\sim 200 \mathrm{ps}$, corresponding to a path length as long as $6 \mathrm{~cm}$ in air. A finer temporal resolution is preferred to resolve tissues' structure better.

In this letter, we propose to measure TPSFs using lowcoherence interferometry, where a temporal resolution smaller than 100 fs can be easily achieved using ordinary low-coherence sources, such as a superluminescent diode, a femtosecond laser, or a wavelength-swept laser. This method has high sensitivity $(90-140 \mathrm{~dB})$ due to the amplification of signal by a strong reference signal. Also, it can provide a wide dynamic range because the interference signal is proportional to the amplitude, instead of the intensity, of the

${ }^{a)}$ Electronic mail: lhwang@biomed.wustl.edu light emitted from the sample. This detection scheme is the basis for OCT.

OCT works in the ballistic regime, where the measured photons are assumed to take straight paths. It is able to provide micron-scale resolution imaging of tissues' scattering structure. However, like other ballistic imaging modalities, its imaging depth is limited to $\sim 1 \mathrm{~mm}$, due to the rapid exponential attenuation of ballistic photons. In a highly scattering medium, the image quality degrades quickly after light penetrates several hundreds of micrometer because of multiple scattering. ${ }^{8}$ The multiple-scattered light can interfere with the reference light just like the single-scattered light. ${ }^{9}$ It is also difficult to extract absorption information from OCT. Spectral information has previously been exploited to solve this problem at the cost of spatial resolution. ${ }^{10}$ However, this method is susceptible to the spectral variation of the scattering coefficient $\mu_{s}$, and is also unable to detect absorbers whose absorption coefficient $\mu_{a}$ does not change much in the source bandwidth.

OCCT bridges the gap between DOT and OCT. It works in the quasidiffusive regime, ${ }^{11}$ where most photons are multiply scattered, however not totally diffused. The sidescattered photons are also collected. It makes full use of the multiple-scattered photons by adopting a DOT-like modelbased reconstruction, and provides a tomographic imaging technique that provides deeper penetration than OCT and finer spatial resolution than DOT. In addition, because the full inverse transient radiative transfer problem is solved during the reconstruction, it can potentially map all the optical properties.

Our OCCT system (Fig. 1) is based on a fiber-optic Mach-Zehnder interferometer. It is seeded by a broadband superluminescent diode (IPSDD0803, InPhenix, center wavelength $\lambda_{0}=829 \mathrm{~nm}$, bandwidth $\Delta \lambda=36.4 \mathrm{~nm}$ ). Therefore, the achievable temporal resolution here is $56 \mathrm{fs}$, which can be further reduced by increasing the source bandwidth. The cross interference between light traveling through a fixed-length reference arm and the sample arm is recorded by a homemade spectrometer using a fast line-scan camera (Aviiva M2, Atmel, 12 bits, 2042 pixels). This spectraldomain detection has been proven to be faster and more sensitive than its time-domain counterpart. ${ }^{12,13}$ The polarization and dispersion differences between the two arms are carefully compensated for. The TPSF is calculated from the re- 


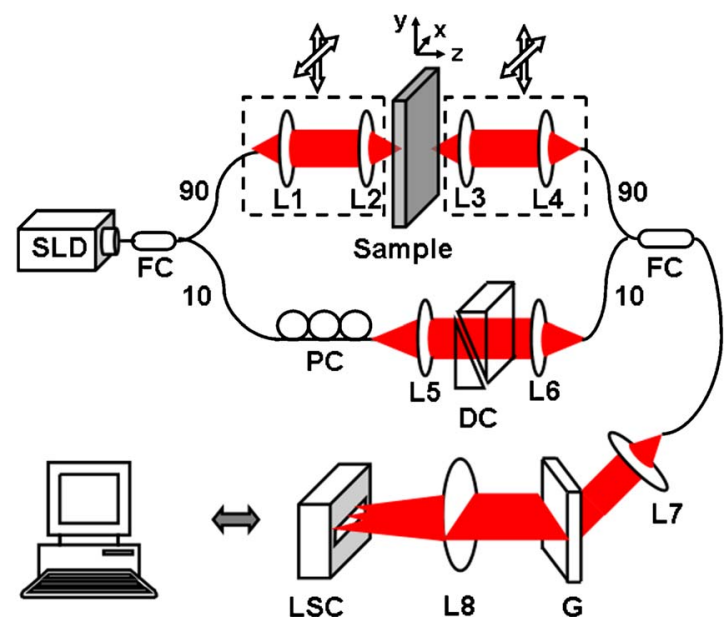

FIG. 1. (Color online) Optical coherence computed tomography (OCCT) system. TPSFs at different source-detection pairs are measured by spectraldomain low-coherence interferometry. SLD: superluminescent diode; FC: fiber coupler; PC: polarization controller; DC: dispersion compensation; G: gratings; LSC: Line-scan camera; L1-L8: lenses. corded spectrum through Fourier transform. The illumination and collection optics are prealigned coaxially. Both are scanned laterally (in the $x-y$ plane) by a translation stage with a step size of $100 \mu \mathrm{m}$. This system can be easily parallelized, for example, using a coherent fiber bundle, in the future to increase the frame rate.

Unlike DOT, OCCT cannot use diffusion theory to model the underlying photon-migration problem because the diffusion approximations are invalid for the early arriving photons. We must resort to the full transient radiative transport equation (RTE). As an initial demonstration of the OCCT concept, we aim to image the spatial distribution of absorption perturbation $\delta \mu_{a}(\mathbf{r})$ here. Perturbations in scattering coefficient $\mu_{s}$ and the scattering phase function can be mapped by following similar procedures. From the RTE, the change in the measurement due to this absorption perturbation within the acceptance angle $\Omega_{d}$ is obtained under the first Born approximation as

$$
\begin{aligned}
\Delta T\left(\mathbf{r}_{d}, t_{d}\right) & =-\int_{\Omega_{d}} \int_{V} \int_{4 \pi} \int_{t} \hat{n}\left(\mathbf{r}_{d}\right) \cdot \hat{s}_{d} S\left(\mathbf{r}_{s}, \hat{s}_{s}, t_{s}\right) G\left(\mathbf{r}, \hat{s}, t ; \mathbf{r}_{s}, \hat{s}_{s}, t_{s}\right) \delta \mu_{a}(\mathbf{r}) G\left(\mathbf{r}_{d}, \hat{s}_{d}, t_{d} ; \mathbf{r}, \hat{s}, t\right) d t d \Omega d V d \Omega_{d} \\
& =-\int_{V} \delta \mu_{a}(\mathbf{r}) \int_{\Omega_{d}} \int_{4 \pi} \int_{t} \hat{n}\left(\mathbf{r}_{d}\right) \cdot \hat{s}_{d} S\left(\mathbf{r}_{s}, \hat{s}_{s}, t_{s}\right) G\left(\mathbf{r}, \hat{s}, t ; \mathbf{r}_{s}, \hat{s}_{s}, t_{S}\right) G\left(\mathbf{r}_{d}, \hat{s}_{d}, t_{d} ; \mathbf{r}, \hat{s}, t\right) d t d \Omega d \Omega_{d} d V \\
& =-\int_{V} \delta \mu_{a}(\mathbf{r}) J_{0}\left(\mathbf{r} ; \mathbf{r}_{d}, t_{d}\right) d V,
\end{aligned}
$$

where $\hat{n}$ is the inward normal of the detection surface. $S$ is the source term, while $G$ is the Green's function solution for the transient RTE. The integration involving $S$ and $G$ can be merged into a single term $J_{0}\left(\mathbf{r} ; \mathbf{r}_{d}, t_{d}\right)$, which physically means the portion of measured signal that has been affected by the perturbation at $\mathbf{r}$. Equation (1) represents a linear inverse problem, which is widely studied in DOT. $J_{0}$, often referred to as the sensitivity function, is calculated through a time-resolved Monte Carlo method. ${ }^{14}$ The experimental boundary condition is also included in our Monte Carlo simulation. The simultaneous iterative reconstruction technique is used to solve $\delta \mu_{a}(\mathbf{r})$, since it, in general, yields better images than the regularized pseudoinverse approach and the algebraic reconstruction technique. In real experiment, we used $M_{0} \Delta T / T_{0}$ instead of $\Delta T$ directly for inversion, in order to make our reconstruction less susceptible to the boundary condition of the object. Here, $T_{0}$ and $M_{0}$ are the unperturbed measurements obtained through experiment and simulation, respectively.

A highly scattering tissue-mimicking phantom was constructed using aqueous suspension of $1 \mu \mathrm{m}$ polystyrene microspheres. The optical properties calculated using the Mie theory were $\mu_{s}=113.6 \mathrm{~cm}^{-1}$ and scattering anisotropy $g$ $=0.90$, whereas the absorption is negligible. The phantom used in our experiment was $2.6 \mathrm{~mm}$ thick, corresponding to $\sim 30$ scattering mean-free-paths. The integration time for recording a single spectrum was $1 \mathrm{~ms}$. The TPSF for a single source-detector pair was the average based on 500 recorded spectra, where averaging alleviated the speckle noise. Figure 2(a) shows the TPSFs detected at the source-detector pairs with lateral shift $x$ ranging from -0.5 to $0.5 \mathrm{~mm}$. The measurements at $x=0,0.2$, and $0.4 \mathrm{~mm}$, after compensation for the depth-dependent decay due to the finite spectral resolution, match the predictions from the Monte Carlo simulation well, as shown in Figs. 2(b)-2(d).
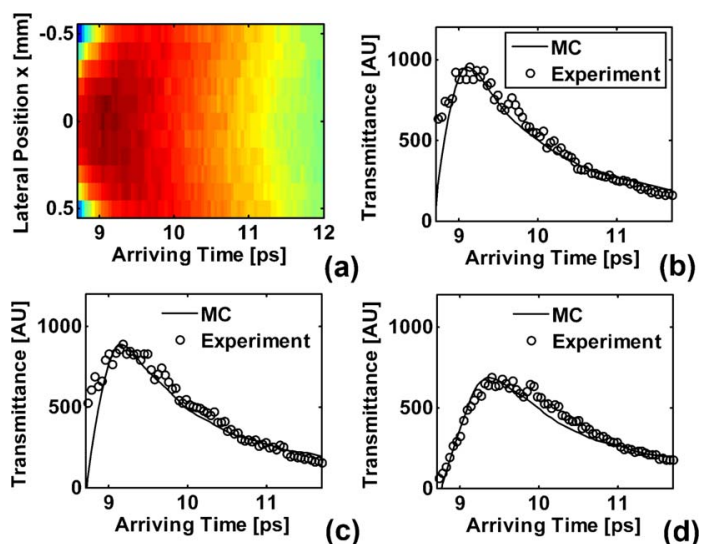

FIG. 2. (Color online) TPSFs measured in OCCT. (a) TPSFs obtained at source-detector pairs with lateral shift $x=-0.5-0.5 \mathrm{~mm}$. TPSFs measured at (b) $x=0 \mathrm{~mm}$. (c) $x=0.2 \mathrm{~mm}$, and (d) $x=0.4 \mathrm{~mm}$ is compared with the predictions from the Monte Carlo simulation. 

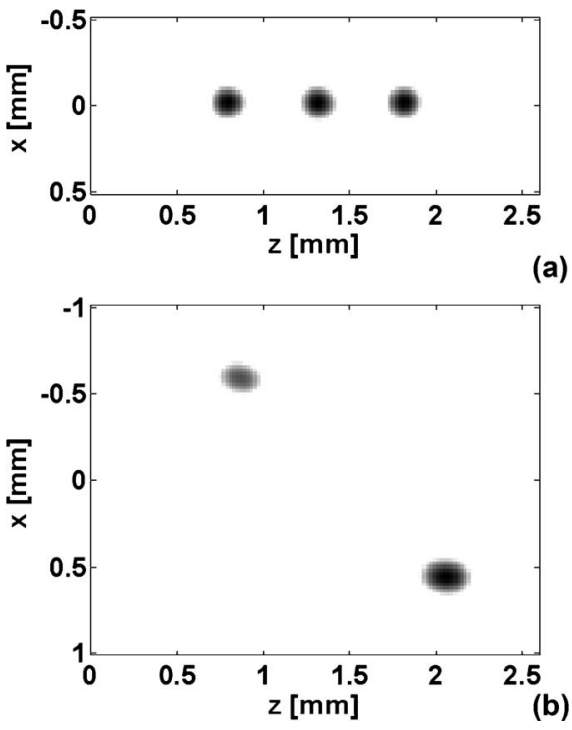

FIG. 3. Reconstructed OCCT images of hair fibers in high-scattering tissue mimicking phantom. (a) OCCT images of a $100 \mu \mathrm{m}$ hair fiber at three different depths with $0.5 \mathrm{~mm}$ separation. (b) OCCT images of two hair fibers with $1.3 \mathrm{~mm}$ separation along both the $x$ and $z$ directions.

A $100 \mu \mathrm{m}$ hair fiber was immersed parallel to the $y$ axis inside the scattering medium described above. Three tomographic images were obtained when this hair fiber was placed at three different depths with a $0.5 \mathrm{~mm}$ separation. For each position, data were collected at $11 \times 11$ source-detector pairs, which covered a $1 \mathrm{~mm}$ range along the $x$ axis. Figure 3(a) shows the combined three reconstructed OCCT images thresholded at $50 \%$ of the peak value in the image. The hair fiber was clearly identified at all three expected positions. The full width at half maximum of the imaged hair is $200 \mu \mathrm{m}$ along the $z$ direction and $180 \mu \mathrm{m}$ along the $x$ direction, both of which are $\sim 100 \mu \mathrm{m}$ wider than the diameter of the hair fiber. Under the assumption of a linear system (a result of the Born approximation), the spatial resolution of our OCCT system is estimated to be $\sim 100 \mu \mathrm{m}$. This resolution maintains well throughout the imaged area. Figure 3(b) shows the reconstructed image of two hair fibers in the scattering medium, with a $1.3 \mathrm{~mm}$ separation in both the $x$ and $z$ directions. Transmitted light was measured at $21 \times 21$ source-detector pairs, which covered a $2 \mathrm{~mm}$ range along the $x$ axis. This demonstrates that OCCT is capable of mapping multiple absorption perturbations simultaneously.

Previous development of imaging modalities for the quasidiffusive regime is limited to laminar optical tomography $(\mathrm{LOT})^{15,16}$, which uses cw measurements. The image quality relies on proper regularization and decays with depth. Since time-resolved measurements are adopted in OCCT, the inverse problem is less ill-posed and the reconstruction is more robust. OCCT is also less sensitive to specular reflections than LOT, since the unwanted reflections fall outside the time gate.

Another unique advantage of OCCT is that it can be scaled up to image thicker tissues by relaxing the temporal resolution because the light detected at each time point increases with increasing time gate. Of course, this is done at the cost of spatial resolution. The scaled-up OCCT is promising to provide a low-cost alternative to the current timedomain DOT. Detection of light after penetrating $1.5 \mathrm{~cm}$ chicken tissue was previously reported by relaxing the time gate to 900 is. 17
The linear perturbation assumption is known to fail when perturbations are large. In this case, the above prediction of spatial resolution would also fail because the system would become nonlinear and the neighboring targets would affect each other's image. Therefore, the nonlinear image reconstruction problem needs to be solved through matching the prediction and the measurement using the forward model iteratively. The current Monte Carlo based forward model, although accurate, suffers from stochastic noise. To achieve acceptable smooth time-resolved simulation for the quasidiffusive regime is extremely computation intensive. As a result, our current experiments are limited to imaging line objects (parallel with the $y$ axis) by using a sensitivity function integrated along the $y$ axis. Thus, a fast forward model is desirable for expanding the application of OCCT. Recently, with the advancement in time-resolved optical image techniques, researchers have started to develop various numerical solutions for solving the full transient RTE based on the discrete transfer method, the discrete ordinates method, and finite volume method. ${ }^{8}$ However, further studies are needed to clarify their applications in the content of biomedical optical imaging, especially for the quasidiffusive regime. Their accuracy need to be tested against the Monte Carlo method, a well-accepted golden standard.

In conclusion, we demonstrated OCCT as a 50-fs-time-resolved tomographic modality to provide tomographical images of absorption perturbations in the quasidiffusive regime. This technique is important for functional studies (e.g., hemodynamics) and molecular imaging based on absorption-modulated probes in human skin and small animals. A reflection-mode OCCT, as well as its scaled-up version and proper fast forward model, is actively pursued in our group.

We thank Dr. Youzhi Li and Dr. Ku Geng for fruitful discussion and insightful suggestions. This work was supported in part by the National Institutes of Health under Grant No. R01 CA092415 and R01 CA106728.

${ }^{1}$ A. Yodh and D. Boas, Biomedical Photonics Handbook (CRC, Boca Raton, 2003), Chap. 21, p. 71.

${ }^{2}$ A. Gibson, J. Hebden, and S. Arridge, Phys. Med. Biol. 50, R1 (2005).

${ }^{3}$ D. Huang, E. Swanson, C. Lin, J. Schuman, W. Stinson, W. Chang, M. Hee, T. Flotte, K. Gregory, C. Puliafito, and J. Fujimoto, Science 254, 1178 (1991).

${ }^{4}$ B. Bouma and G. Tearney, Handbook of Optical Coherence Tomography (Dekker, New York, 2002).

${ }^{5}$ J. Hebden and D. Delpy, Opt. Lett. 19, 311 (1994).

${ }^{6}$ F. Schmidt, M. Fry, E. Hillman, J. Hebdan, and D. Delpy, Rev. Sci. Instrum. 71, 256 (2000).

${ }^{7}$ C. D'Andrea, D. Comelli, A. Pifferi, A. Torricelli, G. Valentini, and R. Cubeddu, J. Phys. D 36, 1676 (2003).

${ }^{8}$ M. Yadlowshy, J. Schmitt, and R. Bonner, Appl. Opt. 34, 5699 (1995).

${ }^{9}$ B. Karamata, M. Laubscher, M. Leutenegger, S. Bourquin, T. Lasser, and P. Lambelet, J. Opt. Soc. Am. A 22, 1369 (2005).

${ }^{10}$ U. Morgner, W. Drexler, F. Kärtner, X. Li, C. Pitris, E. Ippen, and J. Fujimoto, Opt. Lett. 25, 111 (2000).

${ }^{11}$ L. V. Wang and H.-I. Wu, Biomedical Optics: Principles and Imaging (Wiley, New Jersey, 2007), Chap. 5, p. 114.

${ }^{12}$ J. deBoer, B. Cense, B. Park, M. Pierce, G. Tearney, and B. Bouma, Opt. Lett. 28, 2067 (2003).

${ }^{13}$ R. Leitgeb, C. Hitzenberger, and A. Fercher, Opt. Express 11, 889 (2003).

${ }^{14}$ G. Yao and L. Wang, Phys. Med. Biol. 44, 2307 (1999).

${ }^{15}$ A. Dunn and D. Boas, Opt. Lett. 25, 1777 (2000).

${ }^{16}$ E. Hillman, D. Boas, M. Dale, and A. Dunn, Opt. Lett. 29, 1650 (2004).

${ }^{17}$ M. Hee, J. Izatt, E. Swanson, and J. Fujimoto, Opt. Lett. 18, 1107 (1993).

${ }^{18}$ S. Mishra, P. Chugh, P. Kumar, and K. Mitra, Int. J. Heat Mass Transfer 49, 1820 (2006). missions. Download to IP: 131.215.70.231 On: Wed, 06 Jul 2016 